\title{
Towards a Green Supply Chain Based on Smart Urban Traffic Using Deep Learning Approach
}

\author{
Loubna Terrada *, Mohamed El Khaïli , Abdelaziz Daaif , Hassan Ouajji \\ Laboratory SSDIA, ENSET Mohammedia, Hassan II University of Casablanca, Morocco
}

\begin{abstract}
Green Supply Chain Management (GrSCM) has become one of the most crucial innovation in the Supply Chain Management (SCM). This approach involves environmental concerns and issues into the SCM, thus, companies and authorities tend to exploit the GrSCM through logistics process in order to improve their performance. In this paper, we will give a demonstration of the added value of the Urban Traffic Management (UTM) and its integration in the concept of GrSCM, we also aim to study its impact on the performance improvement in Transport Management with a focus on Air quality improvement. This study proposes a new approach and model based on Deep learning for Urban Traffic Control Management to solve the traffic flow problem in order to reduce the congestion, improve the air quality and enhance the urban supply chain. Our proposed framework for Data collection and processing is mainly based on Internet of Thing (IoT) technologies for an efficient Smart City.
\end{abstract}

Keywords Green Supply Chain Management, Environmental Management, Urban Traffic Management, IoT, Smart City, Deep Learning

AMS 2010 subject classifications 92B20, 97R40, 90B06

DOI: $10.19139 /$ soic-2310-5070-1203

\section{Introduction}

Nowadays, the industrial companies tend to enhance their products quality, reduce lead time and costs, taking into account the customer requirements, in line with the changing competitive landscape and pressure on prices, which force them to focus more on sustainability than the environmental issues [1]. The environmental pollution is a serious problem worldwide, knowing that manufacturing and transportation are among the main sources of toxic gas emissions and subsequently affect the scale of air quality. However, Manufacturing industries aim to incorporate Green practices in their Supply Chain Management, which leads us to the concept of Green Supply Chain Management (GrSCM)[2]. Green Supply Chain Management (GrSCM) was introduced as a preventive approach and solution to improve the performance of the company's processes and products. The main aim of this approach is to ensure the economic growth, the environmental sustainability and also to address social and environmental issues. In the other hand, the natural resources and energy exploitation has become irrational and also an international matter and interest among researchers, therefore, the GrSCM emerges as relevant systematic approach and has evolved into a crucial factor across all Supply Chain activities [3]. A. Tomar et al. claims that the research works are insufficient to investigate the integration of GrSCM practices in manufacturing activities,

\footnotetext{
${ }^{*}$ Correspondence to: Loubna Terrada (Email: loubna.terrada@ gmail.com). Laboratory SSDIA, ENSET Mohammedia, Hassan II University
} of Casablanca, Morocco.

ISSN 2310-5070 (online) ISSN 2311-004X (print)

Copyright (c) 2022 International Academic Press 
particularly in countries under development [4]. Our research work deals with Green Supply Chain and Transport, which form an important research field in corporate governance with a particular attention on Urban Traffic Management. An eco-friendly Supply Chain involves efficient management of traffics and roads in Urban zones and a low greenhouse gas emission. Hence, our aim to propose a smart solution of Urban traffic management based on Artificial Intelligence in order to reduce congestion, hence, improve the air quality for an ecological smart city.

In this paper, we will give an overview of GrSCM and urban traffic control to provide a comprehensive picture. Then, we will present the data collection process using IoT technologies. We will also introduce the mathematical model for urban traffic flow and our calculation architecture. In the last sections, we will present the Deep Learning Model used for urban traffic prediction traffic and the project implementation framework in the GrSCM context.

\section{Literature review}

\subsection{Related work on GrSCM}

This paper offers a new framework and model to assess the impact of the Urban Traffic Control Management (UTCM) on the GrSCM. Manufacturing and transport are among the most polluters of environment and that is the main explanation for emerging environmental problems [4]. Furthermore, industries aim to incorporate ecofriendly practices in their SCM so as to attenuate the environmental pollution, thus, Research works are currently led by several activities of the SCM processes in several manufacturing industries in small, medium enterprises and enormous scale manufacturing companies and to review the impact of green practices within the main activities throughout the SCM, i.e. green sourcing, green manufacturing, green warehousing, green distribution, green packaging, green transportation [5, 6].

A study by S. Kumar et al. on the link between GrSCM practices and the ecological performance of companies shows that the performance of the environmentally practices is practiced to make the SCM aligned with international environmental problems due to several stakeholders such as suppliers, customers, employees, investors, the government and to improve the environmental performance [7]. P.Amit et al. studied the impact of GrSCM on the financial and operational performance improvement of industrial companies and showed the relationship between six green approaches, internal environmental management, green supply (procurement), collaboration with customers, investment recovery, green design and reverse logistics [8]. The Survey conducted by S. Yildiz et al. examined the impact of GrSCM on the performance of small, medium and large industries operating in different manufacturing sectors by adopting GrSCM practices, the authors collected data from 159 firms. The Data analysis indicates that the implementation of green practices has a relevant impact on organizational performance, therefore improving the overall performance of the SC from the acquisition of raw materials until the delivery of the final product [9].

In the field of transport, industrial firms tend to implement innovative solutions based on advaced technologies and Artificial Intelligence to predict and tackle environmental problems. In a detailed review undertaken by Sarker et al. the safety, traffic efficiency (i.e. flow control) could be improved by Communication technologies related to the design and implementation of Connected and Automated Vehicles. These technologies can also ensure the cooperation between components of the transport system, e.g. vehicle, transport infrastructure and driver via communication V2V and V2I, thus, improve road safety, air quality, and minimize the energy consumption of the vehicle [10].

According to the reported research works, we summarize the GrSCM performance in three key indicators, as shown in Figure 1. 


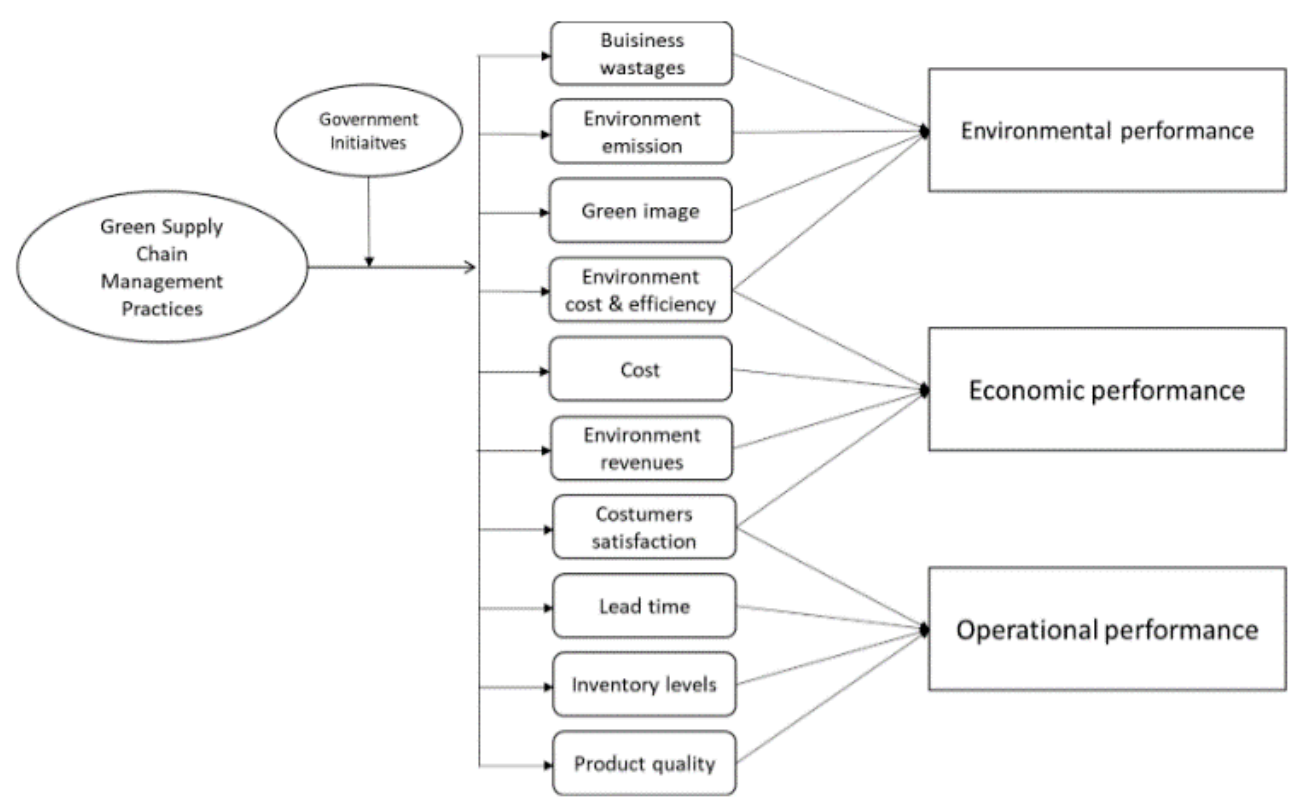

Figure 1. Key Performance Indicators relating to GrSCM

Namely the performance of the company, with regard to environmental performance, economic performance and operating performance $[11,12]$.

\subsection{Urban Traffic Control for Green Supply Chain Management}

The authorities are currently involved in developing environmental solutions to handle the main freight transport issues in industrial zones and cities, such as, congestion, air pollution and noise pollution. Thereby, public authorities collaborate with researcher and practitioners in Urban Logistics to tackle this issue, in accordance with SCM theories and methods for logistics performance measurement [13].

The purpose of GrSCM is to meet the challenges of firms and both public authorities [13, 14], as we summarized in the table that follows:

Table 1. Challenges of Green Urban Transport

\begin{tabular}{|c|c|}
\hline Public Authorities Challenges & Companies Challenges \\
\hline Maintain the economic growth & Provide a high quality service to customers \\
\hline Control the urban development of the City & Reduce transportation costs and the lead time processing \\
\hline Decrease the air pollution rate of the City & Make the transport consistent with the company's sustainable policy \\
\hline
\end{tabular}

In the literature, most studies and models consider congestion as a costly factor, including delays and operating costs, and also make transportation a major cause of global pollution and Greenhouse gas emission, as shown in Figure 2, thereby, GrSCM must be managed through Urban Traffic Management, which aims to control efficiently the transport of goods in urban areas and other traffic flows, in order to achieve the best possible compromise between optimal productivity of the supply system and the environmental concerns[14]. Air pollution is among the most commonly environmental issues of urban areas. because it affects people's health and lifestyle [15, 16]. hence, we aim to propose a smart solution which contribute to the reduction of Greenhouse gas emission based on urban flows prediction according to a Deep Learning approach.

The intelligent environment has emerged as the name of the ecological activities and practices that can reduce the ecological damage; air pollution. They take place in the term "Smart City" involving technologies related to the Internet of Things, other renewable energy sources and also AI methods [17], it also involves traffic control 


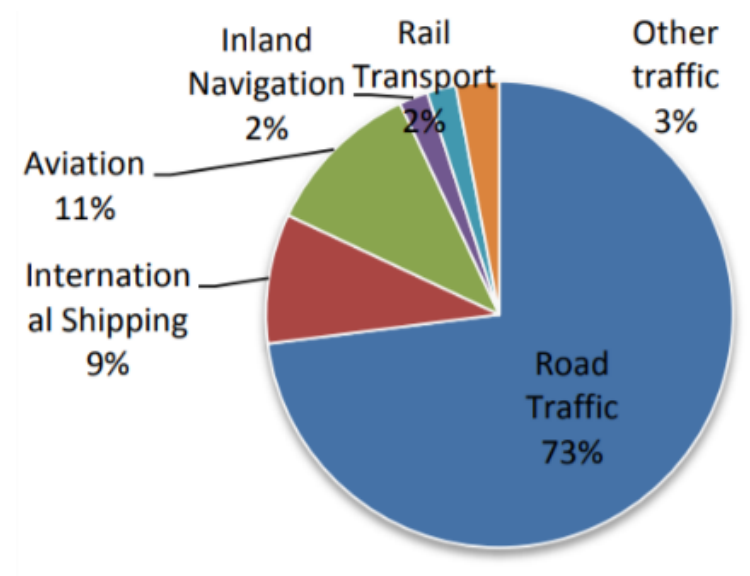

Figure 2. GHG emission from transport sector worldwide[19]

systems in cities, which has a crucial role in managing traffic congestion and minimizing environmental impacts. Conventional techniques for managing traffic signals, such as traffic responsive systems or fixed time weight strategies optimized with historical data, work quite well under normal or expected events [18]. The GrSCM in the context of air pollution is related mostly to the emission of carbon dioxide, knowing that in the urban area, the air pollution due to the direct and indirect emission of this gas. In this respect, a smart ecological city should be equipped with a measurement system of pollution in the air to ensure the urban traffic control. Our research offers a solution to minimize traffic congestion in a city to improve the prediction of road traffic pollution; hence, optimize the flow of crowded road links caused by an emergency road event and help to optimize the light timings in order to help drivers deliver goods on time to the customers or retailers, knowing that transport operators requires solutions in real time to predict and handle unexpected traffic events. These cause huge delays and increased air pollution because of excessive congestion and stationary traffic. The goal of our research work is to propose a model based of Deep Learning prediction from spatial-temporal data in order to provide an accurate forecasts with recurrent and non-recurrent traffic conditions.

\section{Data collection process}

Urban traffic Management required multiple sources that should be processed and prepared for further data analysis. There are various communication system solutions to implement on roads to collect these data, static and dynamic traffic signals give drivers some information about the road when the signals are within visual area. Static signals usually indicate speed limits and various warnings under normal road conditions, while dynamic signals warn drivers of changes in road conditions such as accidents, weather conditions or congestion. From a vehicle perspective, these static and dynamic traffic signals act as lighthouses on the road that transmit specific information to all nearby vehicles. This behavior can be emulated without overloading the main network by implementing a V2I communication network[20].

The V2I network's role is send short messages within its range containing the same information as the current traffic signals, but under the central unit control in real-time. If the vehicle receives real-time alerts about road incidents, they could create dynamic maps to reevaluate and plan their routes to meet some required criteria[20, 21, 22].

Our project is to design objects connected miniaturized and wearables, in order to inform drivers about the air quality in their area. They would be the same as the "tracer activities" to ensure the environment, measurement, which evaluate the efforts and physical performance. This information will be collected and processed in a GIS to help authorities to manage the traffic. We call this new generation of connected objects "enviro-trackers" 
("environmental tracers"). We plan to experiment this solution on the urban area of Greater Casablanca, a city of 4.2 million inhabitants. The air quality measurement is traditionally based on the deployment by the local authority of fixed sensors in the city. Several initiatives have emerged at the intersection of the citizen sensors and the Open Hardware, to design low cost sensors increase coupled smartphones to enable a collaborative data collection (crowdsourcing) and so multiply the observations and measurements as illustrated in Figure 3 [21, 2].

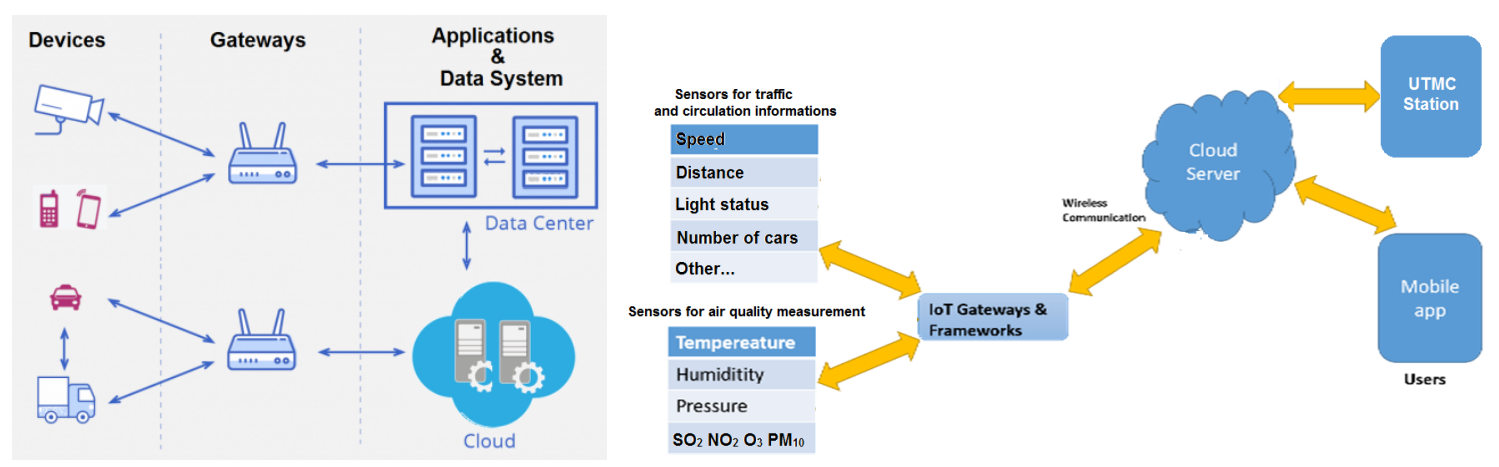

Figure 3. The project deployment

The implementation for data collection requires, the following devices based on IoT architectures [20]:

- Indicators AirParif Method: The index is calculated taking into account the pollutants $\mathrm{SO} 2, \mathrm{NO} 2, \mathrm{O} 3$ and PM10 which are recognized by their health effects.

- Crowdsourcing Architecture: The proposed system is based on a network exchange between a Treatment Unit and multiple Tracers. Each Tracer can transmit data to the Treatment Unit frequently or after a request of the Treatment Unit.

- Sensors: for each pollutant (SO2, NO2, O3 and PM10) to be designed in a chip containing analog and digital blocks to measure the density and the presence of the pollutant emissions. The sensors should be connected to the analog port of the Data Acquisition devices (DAQ device) plus the temperature acquisition as shown in the following Figure 4:

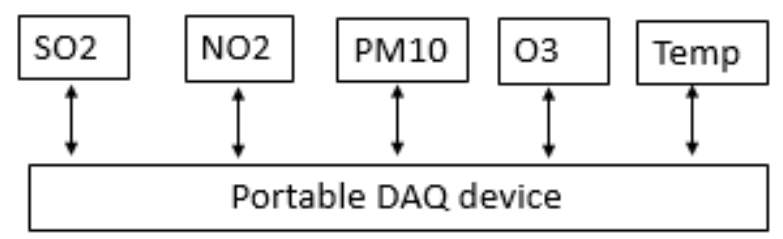

Figure 4. Sensors and DAQ connection

- Wireless technology: wireless communication technologies; Wi-Fi and ZigBee are the primary wireless technologies used for measurement and control systems

- Wi-Fi data acquisition: is an extension of PC-based data acquisition to measurement applications where cables are inconvenient or uneconomical.

- Wireless Sensor Network (WSN) devices: is a wireless network consisting of spatially distributed autonomous device that use sensors to monitor physical or environmental conditions

- Tracers: We propose two type of tracers, static and moving tracers. The static tracers are fixed in facilities like buildings but the moving tracers are fixed on the vehicles or used as application on smartphones of the transporter. The Airparif Air Quality Assessment System is based on four measurements done by SO2 sensor, NO2 sensor, O3 sensor and PM10 sensor. Each tracer is equipped with at least three sensors. 
All tracers should be connected to the Treatment Unit, so that the data could be collected and processed. The index of air quality is computed per each region who correspond to it tracer. The indices can be displayed on a map of the city or used for regulating traffics and other way of management for the city's facilities [18, 20, 21].

Badidi et al. introduce the use of Edge computing which brings data storage and processing closer to the devices that generate and collect the data instead of relying on a data center that can be thousands of miles away[23]. Proximity is essential for applications that require Real-Time data for their operations and very low latency. We can reduce cost by reducing the data volume to be transferred and processed in cloud servers when data are processed locally. Our research work aims to use the spatial-temporal dynamic data for the urban flows prediction, knowing that spatial-temporal data types are widely used in urban flows forecasting and it relates more with our urban crowd flows and traffic flows prediction model.

\section{Mathematical model of the urban traffic flow}

In general, the flow of vehicle arrivals from all the arteries is modeled by a Poissonian flow: if the number of vehicles is a few hundred, the relevance of such modeling is not questionable, at least in terms of first approximation. The possible improvements of this approximation are considered by some authors [24, 25, 26]. Note also that most of the developed algorithms adapt immediately to the case where we replace this Poissonian arrival hypothesis by another arrival law insofar as there is independence of the number of arrivals between $t$ and $t+1$ compared to events prior to time $t$. Now, in a large number of works, it is assumed that the arrivals are of the ON / OFF type. We sometimes say "geometric": between $t$ and $t+1$, there is an arrival of a vehicle with the probability $\alpha(0<\alpha<1)$ and no arrival with the probability $(1-\alpha)$. The models already developed are verified with such a hypothesis and lead to much faster computer processing than those associated with a Poissonian arrivals law.

The question which therefore arises is whether the assumption of ON/OFF arrivals, in the sense above, is a reasonable simplification in order to justify our choice of the type of sources in the remainder of the chapter. There is obviously no universal answer to such a question. To advance the reflection, we will study, in two revealing particular cases, what happens if we replace the Poissonian hypothesis (therefore implicitly assuming that we are in a case where it is indisputable) by the hypothesis of ON/OFF arrivals.

\subsection{Basic process model}

4.1.1. Poissonian traffic model Poisson's law is to traffic models what Bernoulli's law is to models of information sources. Poisson's Law is the most natural and simplest way to describe the process of generating vehicle streams from many independent sources. In general, Poisson's law is considered to be the law of low probabilities [25]. Let us return to the description of the Poisson distribution in urban transport networks. Suppose the vehicle generation rate of a source is $\lambda$ vehicles per unit time, and consider an arbitrary time interval of length $\Delta t$. Poisson's law establishes that the probability that $k$ vehicles ( $k$ integer) are generated during the time interval in question is:

$$
P_{k}=\frac{(\lambda \Delta t)^{k}}{k !} e^{-\lambda \Delta t}
$$

Another way of describing Poisson's law is to characterize the random duration $I$ separating two consecutive events. The probability, $\operatorname{Prob}\{I \leq t\}$, for this duration to be greater than a positive real $t$ given according to the expression:

$$
\operatorname{Prob}\{I \geq t\}=e^{-\lambda t}
$$

Or, on an infinitesimal scale:

$$
\operatorname{Prob}\{I \in[t, t+d t]\}=e^{-\lambda t} \lambda d t
$$

There are several traffic models based on Poisson's law. There is the model of the $n$ Poissonian sources of respective rates $\lambda_{i}$ vehicles per channel. The cumulative rate is:

$$
\lambda=\sum \lambda_{i}
$$


We have also seen the very important model of the infinite population, at cumulative intensity $\lambda$.

4.1.2. Traffic ON / OFF model We will start with the simplest case of the basic model proposed by Pellaumail et al. that we briefly recall [25]. This model is a Markovian process $X$ which evolves in discrete time and which is integer. Its law of evolution is defined by the equation as follows:

$$
X(k+1)=\sup (0, X(k)-1)+A(k)
$$

Where $A(k)$ is an integer random variable independent of $X(k)$ and of fixed distribution.

This $X$ process models the delays of vehicles accessing a fixed flow lane on which there are no other vehicles, the law of $A(k)$ determining the law of the flow of arrivals. At the start, we will study the stationary regime of $X$ in the case where $A(k)$ follows a Poisson law with parameter $\alpha$. We show, for example, that in a steady state, the expectation of $X(k)$ is:

$$
\beta=\alpha+\frac{\alpha^{2}}{2(1-\alpha)}
$$

Let us now consider the case where we suppose that $A(k)$ follows the following ON / OFF law:

$$
\left\{\begin{array}{l}
\operatorname{Prob}\{A(k)=1\}=\alpha \\
\operatorname{Prob}\{A(k)=0\}=1-\alpha
\end{array}\right.
$$

Elementary calculations show that in a steady state, the associated law of $X$ is:

$$
\left\{\begin{array}{l}
\operatorname{Prob}\{X(k)=1\}=\alpha \\
\operatorname{Prob}\{X(k)=0\}=1-\alpha
\end{array}\right.
$$

Pellaumail et al. proposes an algorithm to simulate the flow of traffic on telecommunications networks [24]. They find that convergence towards this stationary regime is very rapid: for applications to ATM networks, it is therefore obviously this stationary regime that plays the most important role. However, we have just noted that the stationary law associated with ON/OFF arrivals is profoundly different from the stationary law associated with Poissonian arrivals. In particular, in the ON/OFF case, the expectation of $X(k)$ tends towards $\alpha$ while in the Poissonian case, this same expectation tends towards $\beta$ which tends towards infinity when $\alpha$ tends towards the value 1 while the mean of the law of arrivals is the same in both cases.

\subsection{Fluid modeling of road network flows}

Recall that in telecommunications, the discrete event simulation of ATM/SDH networks has often been based on models at the burst level. Under this approach the ATM cell forms the elementary level of abstraction "the molecule" as well as the simulation events corresponding to the transit of individual cells through network components. This offers some advantages, but on the other hand the computation time is often too high: for example, simulating traffic on a communication link at $155 \mathrm{MBit} / \mathrm{s}$ for three seconds involves processing about 106 events [25, 26, 27].

Under the fluid approach, the sources of traffic flow are represented by models at the level of "burst-level models"; which makes it possible to use a timescale that is much larger than the passage time of a vehicle. We then consider that the sources do not emit individual vehicles but a continuous flow similar to a fluid that can be described by its instantaneous flow (expressed in vehicles/s).

Although there are many analytical studies of fluid models, there is very little dedicated work on simulations based on these models, and more specifically on traffic simulation of urban road networks. In this section, we describe the equations that govern the operation of the fluid model followed by the principles used to build the complete model to simulate urban road networks [28, 29].

4.2.1. Dynamics of a fluid reservoir Consider a fluid reservoir characterized by a finite capacity B and a limited output flow rate c (constant positive integer). This reservoir is supplied by one or more sources of fluid, the total 
flow rate of which may possibly exceed c. In the following, we will develop the equations describing the dynamic behavior of such a reservoir, in particular with regard to the occupation of the reservoir and the "outflow" process, and that for a particular class of arrival process. This development is carried out in order to apply these equations to the discrete event simulation of a network of fluid reservoirs.

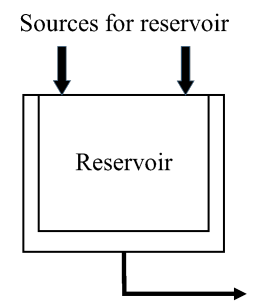

Figure 5. Presentation of a reservoir

4.2.2. Arrival process Let $\Lambda(t)$ be the total flow rate of the fluid entering the reservoir at time $t$; then, the volume of fluid $A\left(t_{a}, t_{b}\right)$ which arrives at the reservoir during the time interval $\left[t_{a}, t_{b}\right]$ is given by:

$$
A\left(t_{a}, t_{b}\right)=\int_{t_{a}}^{t_{b}} \Lambda f(t) \mathrm{d} t
$$

By convention, we denote by $A(t)$ the quantity $A(0, t)$. In general, we will only be interested in arrival processes such that, for each trajectory of $\Lambda$, there is a sequence:

$$
\tau_{A}=\left\{T_{n} \in \mathbb{N} \quad \mid \quad T_{n}<T_{n+1}, n \in \mathbb{N} ; T_{0}=0\right\} .
$$

Such as:

$$
\begin{aligned}
& \Lambda(t)=\Lambda\left(T_{n}\right), \quad \forall t \in\left[T_{n}, T_{n+1}[\right. \\
& \Lambda\left(T_{n}\right) \neq \Lambda\left(T_{n+1}\right)
\end{aligned}
$$

In other words, the trajectories $\Lambda(t)$ must be "stepped" functions, and therefore the trajectories $A(t)$ are continuous and piecewise linear; the sequence of $T_{n}$ corresponds to the dates when $\Lambda(t)$ changes its value.

4.2.3. The fluid amount in the reservoir Let $Q(t)$ be the quantity or "level" of fluid contained in the reservoir at time t. The evolution of $Q(t)$ is described by:

$$
Q(t)=Q(0)+\int_{0}^{t}(\Lambda(s)-c) d s, \quad \forall t \geq 0
$$

With $c$ the maximum outlet flow from the tank.

We define the set $q$, for a reservoir of infinite capacity, as follows:

$$
q=\{s \geq 0 \quad \mid \quad \Lambda(s)>c \quad \text { where } \quad Q(s)>0\}
$$

For a finite capacity tank, it is possible that $Q(t)$ reaches $B$ at a finite instant $t$. So, we must modify the previous expression of $Q$ to satisfy this constraint, which gives:

$$
q=\{s \geq 0 \mid(\Lambda(s)>c \text { and } Q(s)<B) \text { where }(\Lambda(s) \leq c \text { and } Q(s)>0)\}
$$

In this case, the integral in Equation 11 can be interpreted as follows: 

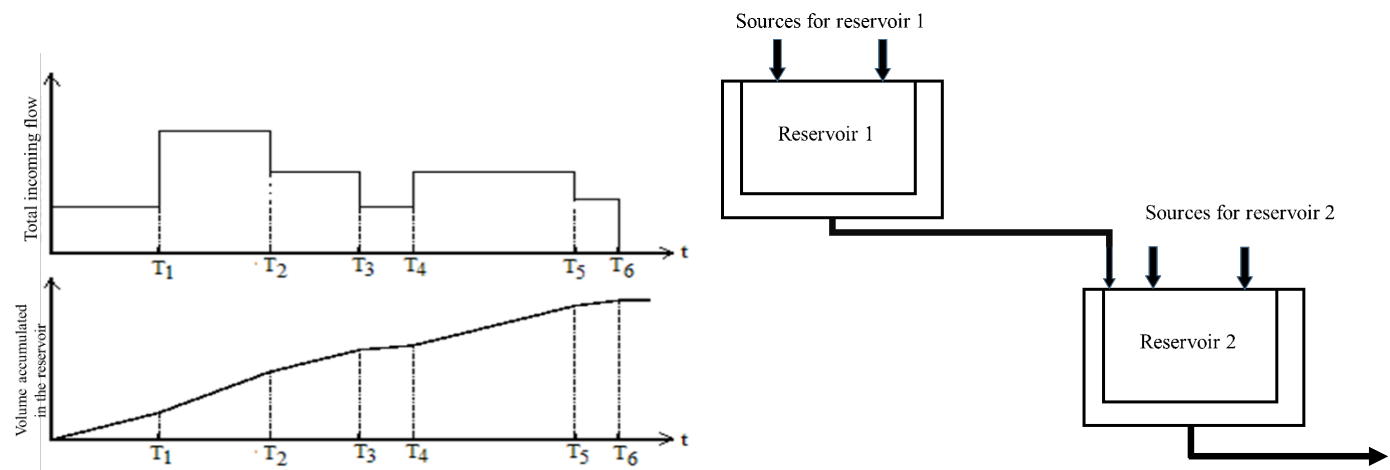

Figure 6. Trajectories of $\mathrm{Q}(\mathrm{t})$ and flow $\Lambda(t)$

- The fluid accumulates at the rate of $\Lambda(t)>c$ units of volume per unit of time, and this during the intervals when the inlet flow exceeds the maximum outlet flow and when the tank is not completely filled (condition 1: $\Lambda(s)>c$ and $Q(s)<B)$

- The tank empties at the rate of $c>\Lambda(t)$ units of volume per unit of time, as long as the inlet flow rate is less than that of the outlet and there is still fluid remaining (condition $2: \Lambda(s)<c$ and $Q(s)>0$ ).

- The condition $\Lambda(s)=c$ and $Q(s)>0$, corresponding to the case where the fluid level remains constant and strictly positive, could be eliminated without changing the value of the integral in Equation 11; however, this condition is included for convenience, since $q$ thus represents the set of time intervals where the reservoir is neither empty nor overflowing.

The resulting trajectories $Q(t)$ are piecewise linear, with slope:

$$
Q(t)=(\Lambda(t)-c), \quad t \in q
$$

The slope changes occur either at the times when the reservoir becomes empty or saturated, or at the dates $T n$ of change in value of $\Lambda(t)$ such as $T_{n}$.

By developing the equation 11 for $t \in\left[T_{n}, T_{n+1}\right.$ [, we obtain :

$$
\begin{aligned}
Q(t) & =Q(0)+\int_{0}^{T_{n}}(\Lambda(s)-c)_{\{s \in q\}} d s+\int_{T_{n}}^{t}+(\Lambda(s)-c)_{\{s \in q\}} d s \\
& =Q\left(T_{n}\right)+\int_{T_{n}}^{t}+(\Lambda(s)-c)_{\{s \in q\}} d s \\
& =\min \left\{B, \quad\left(Q\left(T_{n}\right)+\left(\Lambda\left(T_{n}\right)-c\right)\left(T_{n+1}-T_{n}\right)\right)^{+}\right\}
\end{aligned}
$$

From which we deduce the recurring equation:

$$
\begin{gathered}
Q\left(T_{n+1}\right)=\min \left\{B,\left(Q\left(T_{n}+\left(\Lambda\left(T_{n}\right)-c\right)\left(T_{n+1}-T_{n}\right)\right)\right)^{+}\right\} \\
\text {with }(x)^{+} \equiv \max (0, x)
\end{gathered}
$$

\section{Extreme tank states:}

In addition to the state of the reservoir given by equations 15 or 16 , "extreme" states of the reservoir can be formally defined, linked to the conditions on the flow rates and the accumulated volumes as follows:

- Drying: the tank is empty with a total outflow that exceeds the total inflow. We define $V$ : all the drying times of the tank. 
- Overflow: The tank is full with a total inflow that exceeds the total outflow. We define $D$ : the set of overflowing instants of the reservoir.

- Congestion: The tank is at the overflow threshold at time $t$, if the total inflow equals the total outflow with the quantity in the tank reaches maximum capacity, as shown in Figure 7. We define $C$ as all the instants of reservoir congestion where fluid losses can occur.

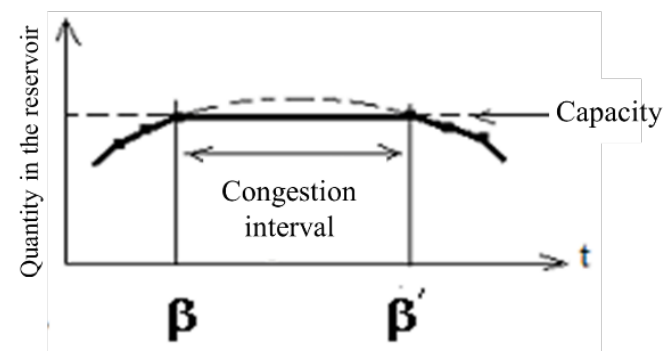

Figure 7. Tank overflow

\section{Definition 1}

The tank is empty at time $t$ if $t \in V$, thus :

$$
V \cong\{s \geq 0 \mid \Lambda(s) \leq c, \text { and }, Q(s)=0\}
$$

Definition 2

The tank is in the overflow (or congestion) state at time $t$ if $t \in C$, thus :

$$
C \cong\{s \geq 0 \mid \Lambda(s)>c, \text { and }, Q(s)=B\}
$$

Definition 3

The reservoir is at the overflow threshold at time $t$ if $t \in D$, thus :

$$
D \cong\{s \geq 0 \mid \Lambda(s)=c, \text { and }, Q(s)=B\}
$$

For $t \in C$, the corresponding congestion period is the interval $\left[\beta(t), \beta^{\prime}(t)[\right.$, such that :

$$
\beta(t) \leq t<\beta^{\prime}(t) \text {. }
$$

Where,

$$
\begin{aligned}
& \beta(t)=\sup \{s<t \mid Q(s)<B, \text { where } \Lambda(s)=c\} \\
& \beta^{\prime}(t)=\inf \{s>t \mid Q(s)<B \text { where } \Lambda(s) \leq c\}
\end{aligned}
$$

It should be noted that $C$ corresponds to the subset of the time when there are losses of fluid; on the other hand, when the reservoir is at the threshold of overflow, there is no loss because the inlet and outlet flow rates are identical. The cumulative fluid losses in the interval $[0, t]$ can therefore be calculated according to:

$$
L(t)=\int_{0}^{t}(\Lambda(s)-c) d s
$$

Dates changes of $Q^{\prime}(t)$ : Let $E_{V}$ be the set of dates of the start of intervals of $V$; similarly, call $E_{C}$ the set of dates of the beginning of the intervals of $C$. Thus, the set of dates of change of $Q^{\prime}(t)$ can be expressed by:

$$
\tau_{Q}=\left(\tau_{a} \cap q\right) \cup E_{v} \cup E_{c} .
$$

For an arbitrary instant $t$, we will denote by $t_{0}(t)$ and $t_{B}(t)$ the following dates:

$$
\begin{aligned}
t_{0}(t) & =\min \left\{s \in E_{v} \mid s \geq t\right\} \\
t_{B}(t) & =\min \left\{s \in E_{c} \mid s \geq t\right\}
\end{aligned}
$$


4.2.4. Departure process Let $R(t)$ be the flow rate of the fluid leaving the reservoir at time $t$. The amount of fluid emitted in the meantime $\left[t_{a}, t_{b}\right]$ is equal to:

$$
D\left(t_{a}, t_{b}\right)=\int_{t_{a}}^{t_{b}} R(t) d t
$$

By convention, we denote by $D(t)$ the quantity $D(0, t)$. Note that the process $R=R(t)$ is such that $R(t) \in[0, c]$, since the maximum flow rate at the outlet of the tank is equal to $c$. In addition, the output flow satisfies the following equation:

$$
R(t)=\left\{\begin{array}{ccccc}
c & \text { if } & Q(t)>0 & \text { where } & \Lambda(t)>c \\
\Lambda(t) & \text { if } & Q(t)=0 & \text { where } & \Lambda(t) \leq c
\end{array}\right.
$$

As $R(t)$ is the sum of the elementary flows leaving also ON/OFF type, we deduce from (23) that $R(t)$ is also a stepped function, continuous to the right; the changes of $R(t)$ taking place at the instants $t \in \tau_{R}$, thus:

$$
\tau_{R}=\left\{t \in \tau_{\Lambda} \mid Q(t)=0\right\} \cup E_{v}
$$

\subsection{Event classes}

The events required for the simulation of a fluid network can be grouped into five main classes:

- Events related to sources; "The flow $\lambda_{i}(t)$ changes".

- Events related to the inflow of reservoirs; "The composition $\lambda_{j}(t)$ changes".

- Events related to the occupation of reservoirs; "The slope $Q_{j}(t)$ changes".

- Events related to the outlet flow of the tanks; "The composition $r_{j}(t)$ changes".

- Events linked to the arrival and departure of discrete objects; "A molecule arrives at reservoir $j$ at time $t$ ".

For each reservoir of the network, the set of times corresponding to the different events is determined as shown in Figures 8 and 9 .

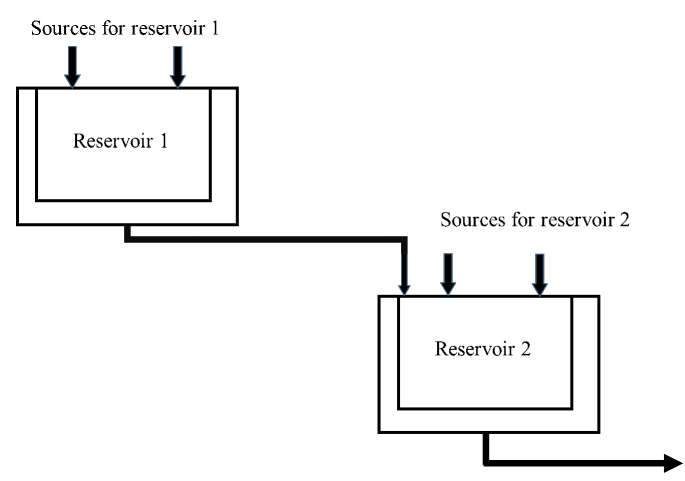

Figure 8. Sets of Reservoirs

Each of these types of events can trigger the generation of others; for instance:

- A class 2 event can trigger one or more class 3 events, spread over time (e. g. " $\lambda_{i}$ goes from $l_{1}$ à $l_{2}$ " can trigger" $Q_{j}^{\prime}$ goes to $q_{1} . . q_{2}$ " and then " $Q_{j}^{\prime}$ goes to $q_{2} . .0$ " );

- A class 3 event can lead to the generation of a class 4 event (the composition becomes equal to $\lambda_{j}$ when $Q_{j}^{\prime}$ becomes zero).

4.3.1. Processing of events To analyze the processing of events during the simulation of a complex network, we will focus on a single reservoir $S$ as shown in Figure 10.

The complex network of reservoirs is supplied by: 


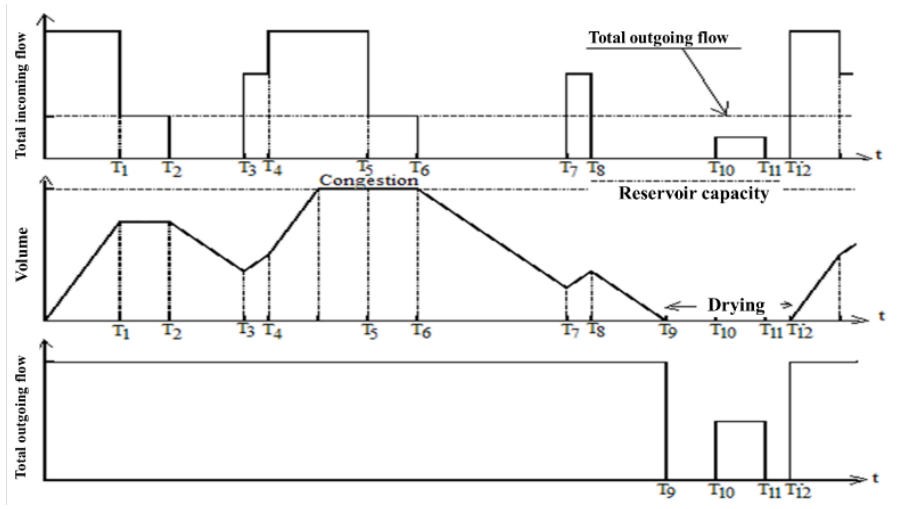

Figure 9. Typical trajectories for $Q(t)$ and Flow $\Lambda(t)$

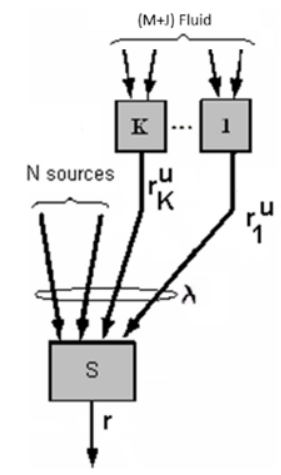

Figure 10. Example of a reservoir in a complex network

- $N$ sources including debits are rated by $\lambda_{1}, \cdots, \lambda_{N}$

- $K$ tanks, whose output rates are rated by $R_{1}^{u}, \cdots, R_{K}^{u}$ and compositions of the outgoing fluid $r_{1}^{u}, \cdots, r_{K}^{u}$. These reservoirs are supplied in total by $M+J \geq K$ individual fluids coming either from other sources or from other reservoirs, and of which only M fluids (numbered $N+1, \cdots, N+M$ ) are poured into $S$.

In addition, in the fluid model of a network, it is also necessary to include " communication links " to link sources and reservoirs. These links introduce a delay in the propagation of rate changes. However, in this section, we will consider all these delays to be zero, without loss of generality. Of course, the reservoir $S$ can itself supply one or more reservoirs. The fluids entering and leaving $S$ are therefore described by:

$$
\begin{gathered}
\lambda(t)=\left(\lambda_{1}(t), \cdots, \lambda_{N}(t), \lambda_{N+1}^{u}(t), \cdots, \lambda_{N+M}^{u}(t)\right) \\
\Lambda(t)=\lambda(t) \cdot e^{T}=\sum_{i=1}^{N+M} \lambda_{i}
\end{gathered}
$$

With $e$ is the unit vector of dimension $N+M$

$$
\begin{gathered}
r(t)=\left(r_{1}(t), \cdots, r_{N}(t), r_{N+1}^{u}(t), \cdots, r_{N+M}^{u}(t)\right) \\
R(t)=r(t) \cdot e^{T}=\sum_{i=1}^{N+M} r_{i}
\end{gathered}
$$


4.3.2. Special features of the schedule The simulator schedule must not only handle the execution of events contained in a tasks list, but also must be able to cancel events already scheduled, before they are executed. In addition, it should be able to deal "simultaneously" with tasks taking place on the same date, for certain classes of events. For example, if $\lambda_{i}(t)$ and $\lambda_{j}(t)(i, j \leq N)$ both change to $t=\tau \lambda(t)$ it is obvious that this should be considered as a single event, from the point of view of $S$; i.e. there should only be one event" the composition $\lambda(t)$ changes to $t=\tau ”$.

4.3.3. Events relating to sources Change in flow : For a source $i$, a discontinuity in the trajectory $\lambda_{i}(t)$ at $t=\tau_{n}$ implies a change in the vector $\lambda(t)$. Therefore, to ensure the synchronization of arrivals in $S$ it is necessary:

- First, process all the simultaneous events relating to components located upstream of $S$, ie execute all the tasks " change the rate $\lambda_{j}(t)$ at $t=\tau_{n}$ and " change the composition $r_{j}^{u}(t)$ at $t=\tau_{n}$ ".

- Then, program a task " $\lambda(t)$ change at $t=\tau_{n} "$.

Generation of Molecules : Discrete fluid molecules " objects can be produced by sources at specific times, to model individual vehicles. These molecules have zero volume (i.e. their arrival at a reservoir does not change its occupancy); in fact, they are considered to be part of the flow produced by the source.

4.3.4. Tank events Change of inlet fluid : An event " $\lambda(t)$ ) changes at $t=\tau_{n}$ " should trigger the following actions:

1. Calculate $Q\left(\tau_{n}\right)$ and $w_{n}$, according to (11) and (16) respectively.

2. Calculate $t_{0}\left(\tau_{n}\right), t_{B}\left(\tau_{n}\right)$ according to (21) and (24) ; then:

- Program an event" $S$ starts to overflow at $t=t_{B}\left(\tau_{n}\right)$ " if this has not already been done and $t_{B}\left(\tau_{n}\right)<\infty$.

- Program an event " $S$ empties at $t=t_{0}\left(\tau_{n}\right)$ " if this has not already been done and $t_{0}\left(\tau_{n}\right)<\infty$.

3. If $w_{n} \neq t_{0}\left(\tau_{n}\right)$, program the task "change $r(t)$ tot $=w_{n} "$.

However, the calculation of $t_{0}\left(\tau_{n}\right)$ and $t_{B}\left(\tau_{n}\right)$ and consequently the decision to program or not an event “ $S$ empties" or " $S$ overflows" has practical problems, because it is necessary to know the 'evolution of $\lambda(t)$ from $t=\tau_{n}$, therefore the evolution of all the components of $\lambda(t)$. However, there is a solution that is easier to implement, based on the following rules:

- Rule 1: A change in the inlet flow at time $t=T_{n}$ is a potential source of overflow if $\Lambda\left(T_{n}\right)>c$ to which corresponds a potential overflow date.

- Rule 2: A change in the inlet flow at $t=T_{n}$ is a potential source of dewatering of the reservoir si $\Lambda\left(T_{n}\right)<c$ to which corresponds a date of potential dewatering.

In addition, note that neither $R(t)$ nor $r(t)$ change during an overflow; therefore, it is not necessary to program an event " $S$ overflows", because it is possible to calculate a posterior volume of fluid lost. For an efficient event cancellation, from the point of view of algorithmic complexity, the reservoir can keep a pointer to the corresponding task which is placed in the schedule list. This way we can extract the event without having to browse the list. Figure 9 shows a typical trajectory for $Q(t)$ and flow $\Lambda(t)$, together with expected overflow and dewatering times; the curves correspond to the parameters: $c=1$ and $B=1$.

Drying of the tank: An event " $S$ is empty at $t=t_{v 0}\left(\tau_{n}\right)$ " triggers the following actions:

1. Make $Q(t)=0$.

2. Program the task"change $r(t)$ at $t=t_{v 0}\left(\tau_{n}\right)$ ", i.e. " now".

Changing the output flows: An event " $r(t)$ changes at $t=w_{n}$ " must occur the following actions:

1. Calculate $r(t)$.

2. Program tasks " $\Lambda(t)$ changes at $t=w_{n}$ " for tanks supplied by $S$.

Action 2 should be synchronized with others (if necessary) to ensure the generation of a single "change of input fluid" event for each of the network components supplied by $S$.

Arrival of a molecule: An event "a molecule arrives at $t=a_{n}$ ", with $a_{n} \in\left[T_{m}, T_{m+1}[\right.$, should be treated as follows: 
1. If $t_{d B}\left(\tau_{m}\right)<a_{n}$ then $S$ is overflowing, so the molecule can be destroyed with a probability which corresponds to the proportion of fluid lost compared to the fluid that arrives.

2. If the molecule is not destroyed, then:

- Calculate the waiting time $W\left(a_{n}\right)$.

- Program the task "a molecule arrives at $t=a_{n}+W\left(a_{n}\right)$ " for the component of the network, located downstream, which must receive the molecule.

\section{Computing model based on Deep Learning}

Nowadays, Deep Learning is widely applied in many fields with great success, such as computer vision, natural language processing, supply chain, power management, and Artificial Intelligence. Deep Learning is different to Machine Learning methods. It demonstrates powerful information extraction and processing capabilities based on massive computation resources. The Deep Learning expands greatly the edge computing applications in various scenarios, improving performance, efficiency and management $[23,30]$.

The typical Deep Learning models are Restricted Boltzmann Machine RBM, AutoEncoder AE, Deep Neural Network DNN, Convolutional Neural Network CNN, Recurrent Neural Network RNN, and Deep Reinforcement Learning DRL as shown in Figure 11 [30]. Understanding the mobility patterns of the vehicles and people is

(a)

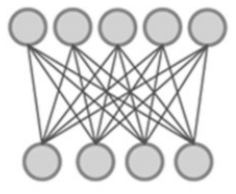

Restricted Boltzmann Machine

(d)

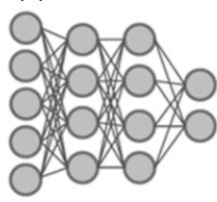

Deep Neural Network

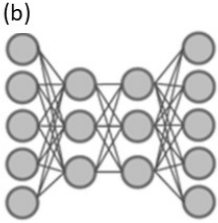

Autoencoder

(e)

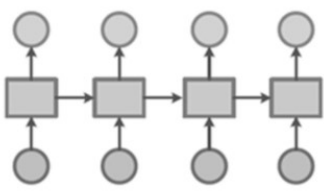

Recurrent Neural Network (c)

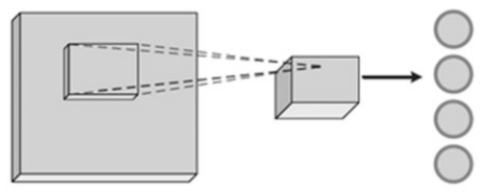

Convolutional Neural Network

(f)

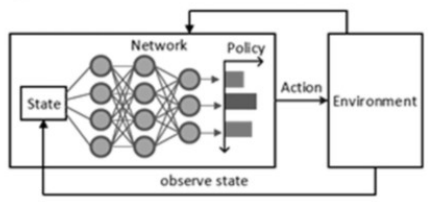

Deep Reinforcement Learning

Figure 11. Structures of different Deep Learning models

a critical problem for urban traffic management. Knowing the distributed features of mobile edge servers; edge computing is naturally ideal for vehicle traffic analysis and prediction [23]. Traditional approaches mostly used time-series analysis or probabilistic graph analysis, which may not sufficiently capture the hidden spatio-temporal relationships therein. As a powerful learning tool, deep learning stands out as an effective method in this direction. Many authors further pointed out the potential of applying different deep learning approaches in urban traffic prediction. They leveraged deep neural network to mine the short term characteristics of the traffic situation of a road segment to predict the near future traffic pattern. A Stacked AutoEncoder (SAE) is used to learn the generic traffic features from the historical data. The impact of weather on traffic situations is also considered and the weather information is incorporated into a deep belief network for integrated learning.

Long Short-Term Memory LSTM model is used for future movement prediction. Huaxiu Yao et al. [31] reviewed the Spatial-Temporal relationships in traffic patterns and proposed a novel Spatial-Temporal Dynamic Network 
STDN based on CNN and LSTM, which outperforms the existing prediction methods. The architecture of STDN consists of the following elements illustrated in Figure 12 :

- Periodically shifted attention mechanism captures the long-term periodic dependency and temporal shifting. For each day, LSTM is used to capture the sequential information.

- The short-term temporal dependency is captured by one LSTM.

- The flow gating mechanism tracks the dynamic spatial similarity representation by controlling the spatial information propagation; "FC" means Fully Connected layers and "Conv" means several Convolutional Layers.

- A unified multi-task prediction component predicts two types of traffic volumes simultaneously.

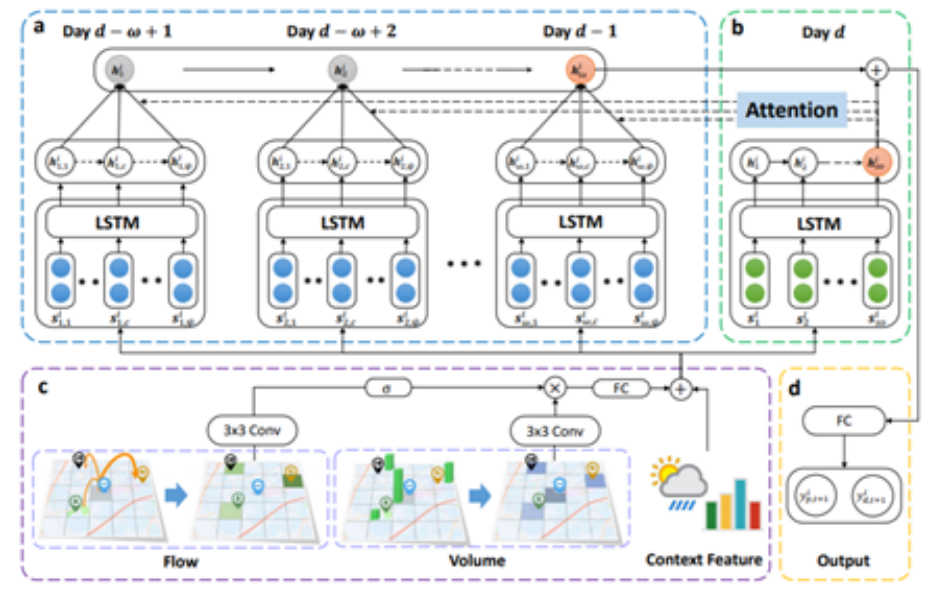

Figure 12. Architecture of Spatial-Temporal Dynamic Network [31]

The architecture proposed by Huaxiu Yao et al. is very interesting. We will adopt it as a calculation model and the LSTM will be adapted to our context of the field study. Figure 12 shows the architecture of our proposed method.

Local Spatial-Temporal Network LSTN (Figure 12(a))

In order to capture the spatial and temporal sequential dependence, the combination of local CNN and LSTM has shown excellent forecasting performance especially in the case of taxi demand prediction approuved by Yao works. Short-term spatial and temporal dependence is managed by combining local CNNs and LSTMs. In order to mutually reinforce the prediction of two types of traffic volumes (i.e. start and end volumes), we integrate and predict them together. This part of our proposed model is called the local space-time network (LSTN).

\section{Local Spatial Dependence}

CNN's network is used to capture spatial interactions. Suggested by Yao et al., Treating the whole city as a picture and just applying a CNN network may not get the best performance. The inclusion of regions with weak correlations with the prediction of a target region negatively impacts performance. So, we use the local CNN to model the spatial dependency.

Short-Term Time Dependency (Figure 12 (b))

We use the Long/Short Term Memory network (LSTM) to capture the temporal sequential dependence, which is proposed to solve the problem of saturation and disappearance of the traditional recurrent neural network (RNN) gradient. Huaxiu Yao et al. [31] used the original version of LSTM (Hochreiter and Schmidhuber 1997).

Spatial Dynamic Similarity (Figure 12 (c))

As we described earlier, local CNN is used to capture spatial dependency. CNN manages the similarity of the local structure by local connection and weight sharing. In local CNN, local spatial dependence is based on the similarity of historical traffic volume. However, the spatial dependence of the volume is stationary, which cannot fully reflect the relationship between the target region and its neighbors. A more direct way to represent interactions between regions is traffic flow. If there is more flow between two regions, the relationship between them is stronger 
(i.e. they are more similar). Traffic flow can be used to explicitly control the propagation of volume information between regions. Therefore, we design a Flow Gate Mechanism (FGM), which explicitly captures the dynamic spatial dependency in the hierarchy.

Similar to local CNN, the local spatial flow image is constructed to strengthen the spatial dependence of the flow. The traffic flow related to a certain region in a time interval falls into two categories, namely, an incoming flow from another location ending in the region during the time interval, and an outgoing flow at from this region to another location. Two flow matrices for the region in this time interval can be constructed accordingly, each item indicating flow in from another region or out to another.

At each layer, we use the flow information to explicitly capture the dynamic similarity between regions by constricting the spatial information through a flow gate. More precisely, the output of each layer is the spatial representation $Y_{i}(k t)$ modulated by the flow gate. After $K$ gated convolution layers, we use a flattened layer followed by a fully connected layer to get the flow-controlled spatial representation as $Y_{i}(t)$. We replace the spatial representation $Y_{i}(t)$ by $\hat{Y}_{i}(t)$. Figure $12(\mathrm{~d})$.

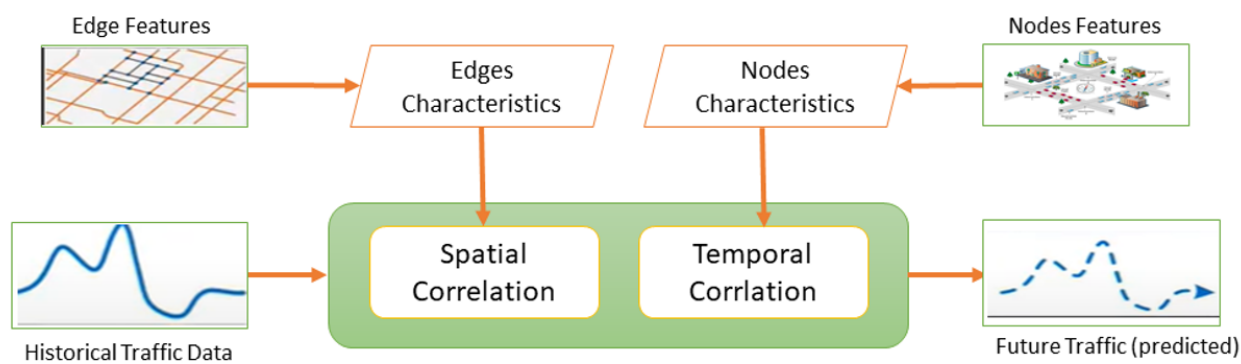

Figure 13. Insight of LSTM Architecture for our UTM solution

The characteristics of locations and their relationships are various. for an efficient urban traffic control in Smart Cities, we adopt a LTSM based mthod which combine Spatial-temporal models as shown in Figure 13. we list below the main Spatio-temporal correlations attributes:

- Nodes attributes : surrounding networks around locations.

- Edge attributes : road connectivity and distances between nodes.

\section{Project implementation}

We worked on a path planning approach in the presence of moving objects[32]. This technique could not give satisfaction since it will not be able to deal with the constraints of traffic in an urban environment. The proposed Urban Traffic Management and Control (UTMC) is based primarily on road junctions in four directions, each of which includes two lanes (go straight, turn right, and turn left) and roads connecting intersections as illustrated in Figure 14.

Each lane is equipped with two basic sensors: the first is located near the traffic light to count the number of vehicle departures and the second established at a variable distance depending on the maximum time permitted for the green light from the first sensor to detect vehicle arrival $[18,2]$. Each traffic light controller describes a time called a cycle, which is a series of phases, and each phase has a green light time required for two simultaneous movements. The following figure illustrates the main layers of the proposed intelligent urban transport architecture.

All these sensors communicate and transmit the traffic information to the UTMC station which calculates the length of the queues for each direction and their average waiting time to check the traffic flow. Depending on the air quality in the area, the UTMC station can extend or shorten light cycles and control dynamic signage. For example, the temporary ban on lane will reduce traffic to improve air quality and transporters will be informed 


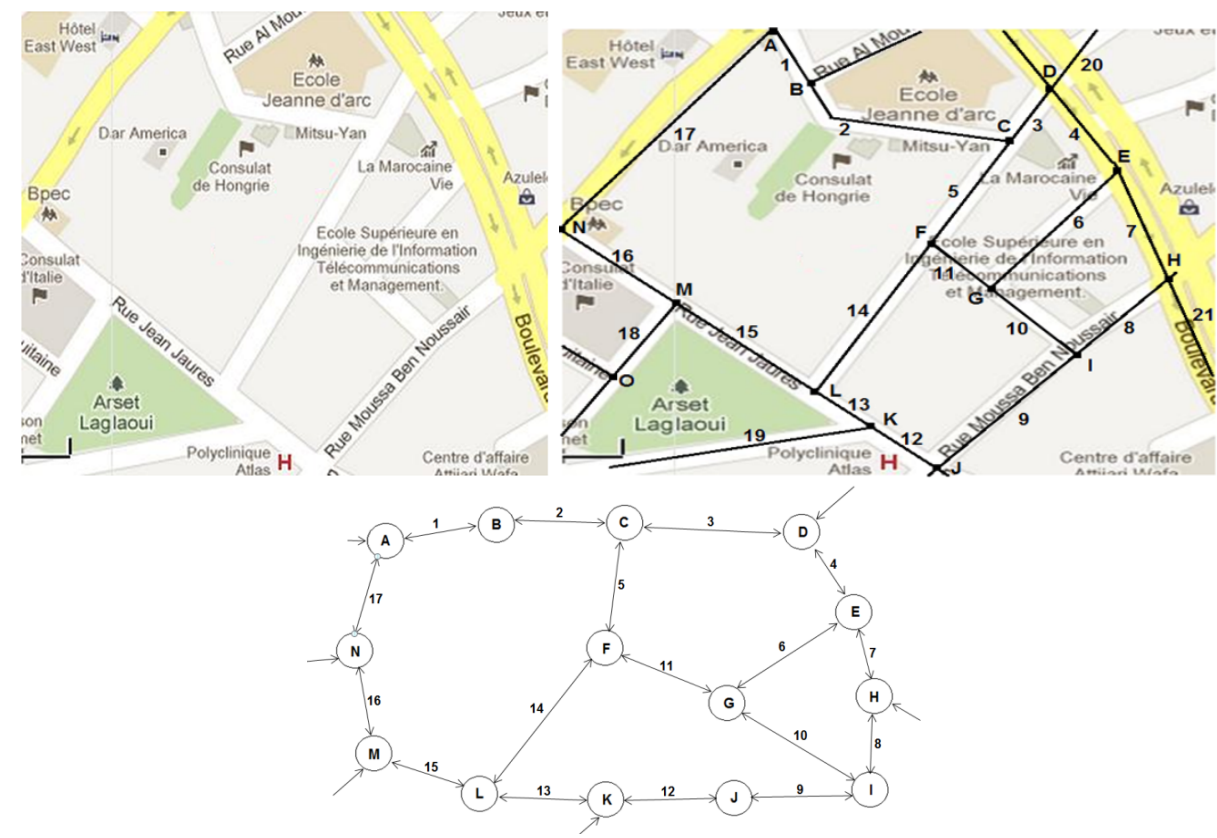

Figure 14. Road map : An oriented graph representation from the map

about alternative routes to reach their destinations as soon as possible to deliver goods on time. We illustrate this process in Figure 15.

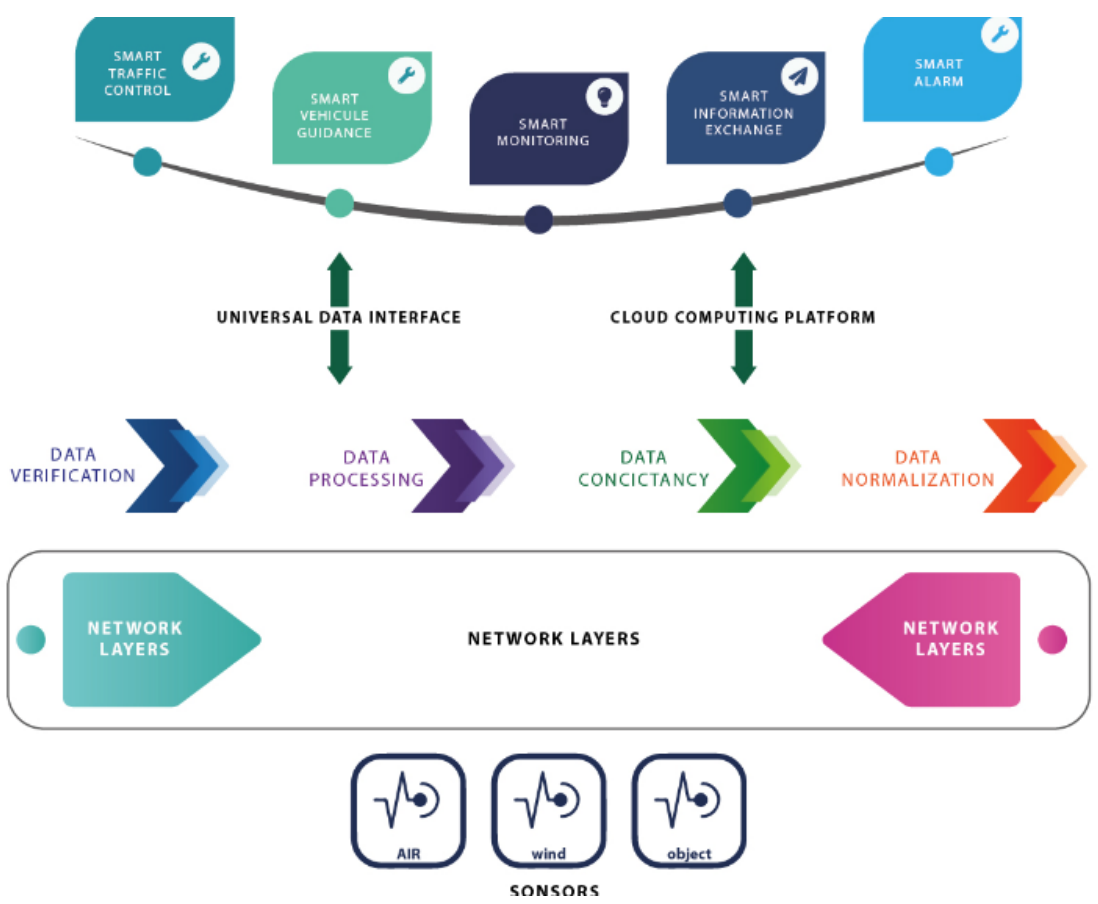

Figure 15. Smart Urban traffic management system architecture based on Cloud Computing and IoT for Data collection and processing 
The main challenge with implementing measurement control systems is that these measures can prove to be ineffective. Trustworthy metering can only involve a congestion transfer from the inside to the outside of the area to be controlled without a change in total travel time and air quality improvement or other traffic congestion effects (simplifies access for urgent deliveries to final customers) . In addition to information about the desired route using a radio broadcast or road signs, a speed warning can be offered as a driving assistance service given the impact on energy consumption and traffic. However, information based on city speed announcements can be based on drivers' experiences of stopping at traffic lights as little as possible, as shown in the following Figure 16.

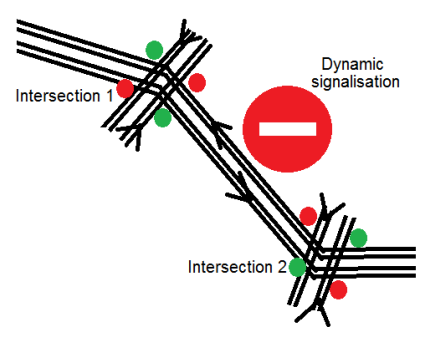

Figure 16. Road Intersections relating to UTMC

The main idea is entirely simple: a definite speed is recommended to drivers to change from light to green at the next intersection. The easiest way to do this is to place an electronically controlled time sign on the side of the road about 100 meters after the previous traffic light, as shown in the Figure 17.

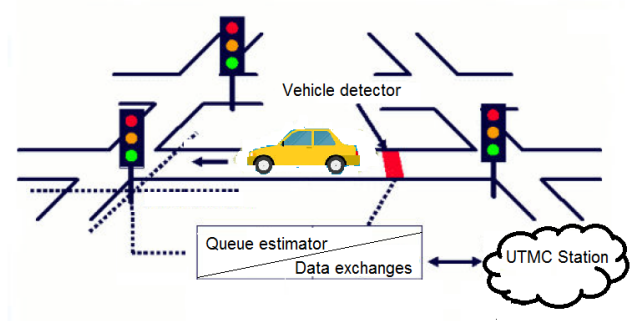

Figure 17. Exchange of information

As shown in Figure 18 and 19, the panel is connected to the lights above and knows how many seconds will pass before they turn green, the electronic controls compute and show a suitable speed to maintain. The UTMC station registers information on the transporters routes and reactions to build a knowledge base to improve the system predictability.

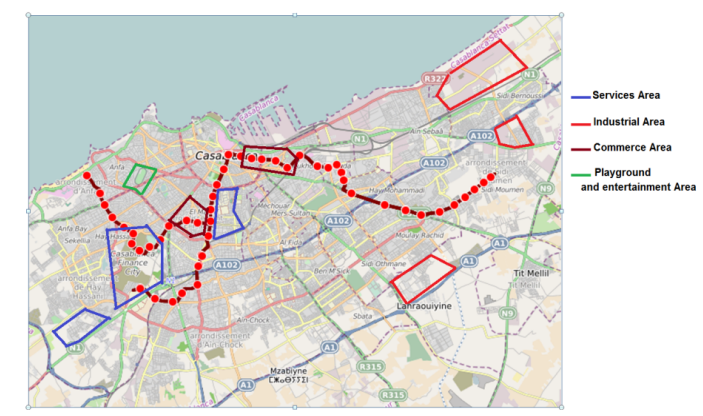

Figure 18. Casablanca traffic network and zone mapping 


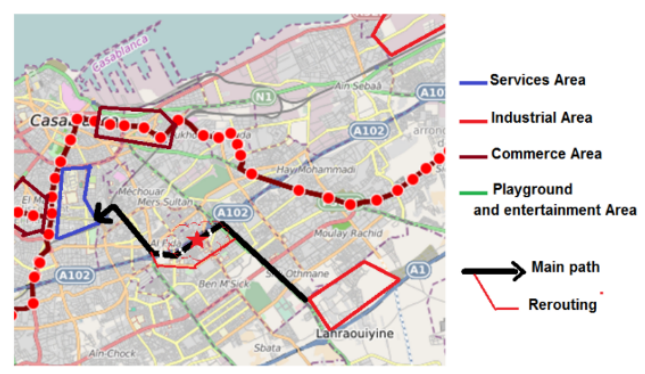

Figure 19. Incident in the main path

Efficient urban planning with a low rate of greenhouse gas emissions is relevant. The process must take into account the factors that influence the level of greenhouse gases in the air, as well as the relationships between the factors and the level of emissions. To do this, we need to:

- Identify the main source of greenhouse gas emissions in the context of green urban traffic.

- Identify components of carbon footprint and determine their importance for using appropriate prevention methods.

- Examine the relationship between carbon footprint and design parameter.

\section{Conclusion and perspectives}

Our approach for Urban Traffic Control Management is based on Artificial Intelligence and IoT, in order to tackle a worldwide issue which is air pollution in cities which means hazards for health and worse quality of life. In the context of urban Logistics, we proposed Mathematical models of route flows and crossroads, to minimize congestion, optimize traffic flow management, thus, reduce the effect of environmental and air pollution. Traffic signalization control has been presented meanwhile the mechanism to control cars which is also needed. While the reported tests are encouraging, a more thorough evaluation is required to better assess real world potential. We also presented the Deep Learning Model adopted for urban traffic prediction.

This study shows that Urban Traffic Control Management is related to the concept of Green Supply Chain, whose strategy is to manage urban freight transport and other traffic flows by integrating green practices for a Smart and Ecological City. As a perspective of our research, we aim to get a DataSet and use LabVIEW Graphical programming as decision support for the Smart City.

\section{Acknowledgements}

We would like to thank the referee for carefully reading our paper and for giving us constructive comments and suggestions to improve our manuscript.

\section{REFERENCES}

1. Loubna Terrada, Jamila Bakkoury, Mohamed El Khaili, and Azeddine Khiat. Collaborative and communicative logistics flows management using the internet of things. In First International Conference on Real Time Intelligent Systems, pages 216-224. Springer, 2017.

2. Loubna Terrada, Mohamed El Khaïli, Hassan Ouajji, and Abdelaziz Daaif. Smart urban traffic for green supply chain management. In 2020 IEEE 2nd International Conference on Electronics, Control, Optimization and Computer Science (ICECOCS), pages 1-6. IEEE, 2020.

3. P. Rao and D. Holt. Do green supply chains lead to competitiveness and economic performance? International Journal of Operations \& Production Management, 25(9):898-916, September 2005. 
4. Anita Tomar and H. Oza. Review of gscm studies relating to green supply chain management practices and performance. AbhinavInternational Monthly Refereed Journal Of Research In Management \& Technology, 5:49-55, 2016.

5. Samir K. Srivastava. Green supply-chain management: A state-of-the-art literature review. International Journal of Management Reviews, 9(1):53-80, 2007.

6. A. K. Bhetja and R. Babbar. Study of green supply chain management in the indian manufacturing industries: A literature review cum an analytical approach for the measurement of performance. 2011.

7. Sanjeev Kumar, Somnath Chattopadhyaya, and Vinay Sharma. Green supply chain management: A case study from indian electrical and electronics industry. International Journal of Soft Computing and Engineering, 1(6):275-281, 2012.

8. R PandyaAmit and M Mavani Pratik. An empirical study of green supply chain management drivers, practices and performances: with reference to the pharmaceutical industry of ankleshwar (gujarat). IJEMS, 3(3):339-355, 2012.

9. Sibel Yildiz Çankaya and Bulent Sezen. Effects of green supply chain management practices on sustainability performance. Journal of Manufacturing Technology Management, 2019.

10. Ankur Sarker, Haiying Shen, Mizanur Rahman, Mashrur Chowdhury, Kakan Dey, Fangjian Li, Yue Wang, and Husnu S Narman. A review of sensing and communication, human factors, and controller aspects for information-aware connected and automated vehicles. IEEE transactions on intelligent transportation systems, 21(1):7-29, 2019.

11. Meeta Gandhi and Hari Vasudevan. Green supply chain management practices and its impact on business performance. In Proceedings of International Conference on Intelligent Manufacturing and Automation, pages 601-611. Springer, 2019.

12. Terrada Loubna and Alloubane Abdelkarim and Bakkoury Jamila and El Khail Mohamed IoT contribution in supply chain management for enhancing performance indicators 2018 International Conference on Electronics, Control, Optimization and Computer Science (ICECOCS), pp 1-5, IEEE, 2018.

13. Joëlle Morana. Sustainable supply chain management in urban logistics. In Sustainable urban logistics: Concepts, methods and information systems, pages 21-35. Springer, 2014.

14. Agata Mesjasz-Lech. Urban air pollution challenge for green logistics. Transportation Research Procedia, 16:355-365, 2016.

15. Maria-Lluîsa Marsal-Llacuna, Joan Colomer-Llinàs, and Joaquim Meléndez-Frigola. Lessons in urban monitoring taken from sustainable and livable cities to better address the smart cities initiative. Technological Forecasting and Social Change, 90:611$622,2015$.

16. Shivaji Bhandarkar. Vehicular pollution, their effect on human health and mitigation measures. VE, 1(2):3340, 2013.

17. Soumaya Ben Letaifa. How to strategize smart cities: Revealing the smart model. Journal of business research, 68(7):1414-1419, 2015.

18. Mohamed El Khaili, Abdelkarim Alloubane, Loubna Terrada, and Azeddine Khiat. Urban traffic flow management based on air quality measurement by iot using labview. In The Proceedings of the Third International Conference on Smart City Applications, pages 193-207. Springer, 2018.

19. Mengpin Ge, Johannes Friedrich, and Leandro Vigna. 4 charts explain greenhouse gas emissions by countries and sectors. 2020.

20. Azeddine Khiat, Ayoub Bahnasse, Jamila Bakkoury, Mohamed El Khaili, and Fatima Ezzahraa Louhab. New approach based internet of things for a clean atmosphere. International Journal of Information Technology, 11(1):89-95, 2019.

21. Mohamed El Khaili, Jamila Bakkoury, Azeddine Khiat, and Abdelkarim Alloubane. Crowdsourcing by iot using labview for measuring the air quality. In Proceedings of the 3rd International Conference on Smart City Applications, pages 1-8, 2018.

22. Abdelaziz Daaif, Mohamed Youssfi, Omar Bouattane, and Oum El Kheir Abra. An efficient distributed traffic events generator for smart highways. (IJACSA) International Journal of Advanced Computer Science and Applications, 8(7), 2017.

23. Elarbi Badidi, Zineb Mahrez, and Essaid Sabir. Fog computing for smart cities' big data management and analytics: A review. Future Internet, 12(11):190, 2020.

24. Jean Pellaumail, Pierre Boyer, and Patrice Leguesdron. Réseaux ATM et P-simulation. Hermès, 1994.

25. Jean Pellaumail. Graphes, simulation, 1-matrices. 1992.

26. Zoubida Jadda and Jean Pellaumail. Basic processes in atm networks. Journal of Pure and Applied Algebra, 146(2):185-196, 2000.

27. José Incera, Raymond Marie, David Ros, and Gerardo Rubino. Fluidsim: A tool to simulate fluid models of high-speed networks. In International Conference on Modelling Techniques and Tools for Computer Performance Evaluation, pages 230-246. Springer, 2000.

28. VV Dixit. Expected utility derivations of two-fluid model for urban traffic. Transportation Research Part C, 35:115-126, 2013.

29. Elizabeth G Jones and Wahid Farhat. Validation of two-fluid model of urban traffic for arterial streets. Transportation research record, 1876(1):132-141, 2004.

30. Fangxin Wang, Miao Zhang, Xiangxiang Wang, Xiaoqiang Ma, and Jiangchuan Liu. Deep learning for edge computing applications: A state-of-the-art survey. IEEE Access, 8:58322-58336, 2020.

31. Huaxiu Yao, Xianfeng Tang, Hua Wei, Guanjie Zheng, and Zhenhui Li. Revisiting spatial-temporal similarity: A deep learning framework for traffic prediction. In Proceedings of the AAAI conference on artificial intelligence, volume 33, pages 5668-5675, 2019.

32. Mohamed El Khaili. Path planning in a dynamic environment. Int. J. Adv. Comput. Sci. Appl, 1(5):86-92, 2014. 